\begin{tabular}{|c|c|c|c|}
\hline \multirow{2}{*}{$\begin{array}{l}\lambda \\
\AA\end{array}$} & \multicolumn{2}{|c|}{ Transparenz } & $\begin{array}{l}\text { Transparenz- } \\
\text { verhältnis }\end{array}$ \\
\hline & Stufe I & Stufe II & $\mathrm{I} / \mathrm{II}$ \\
\hline 4365 & $54,3 \% \pm 0,5$ & $11,3 \% \pm 0,3$ & 4,80 \\
\hline 3655 & $54, \overline{3}$ & $11, \overline{4}$ & 4,76 \\
\hline 3132 & 53,2 & 10,8 & 4,93 \\
\hline 2537 & 50,3 & 65 & 7,73 \\
\hline
\end{tabular}

Tab. 2. Eichung des Stufenfilters.

$3300 \AA$ in Übereinstimmung mit den vorliegenden Messungen gefunden, die erst bei etwa $3100 \AA$ ein merk- liches Absinken der Transparenz zeigen. Messungen von G. Han se ${ }^{8}$ (Zeißwerk Jena) weisen dagegen bei etwa $5000 \AA$ ein Minimum auf, dessen Tiefe und genauer Ort von der Schichtdicke abhängig sind. Die Diskrepanz dieser Ergebnisse zeigt wiederum die Notwendigkeit einer sorgfältigen Eichung solcher Filter in jedem einzelnen Falle.

Die vorliegende Methode wurde im Winter 1945/46 ausgearbeitet. Dem Leiter der Forschungsstelle, Hrn. Erich Regener, bin ich für stete Förderung der Arbeit zu herzlichem Dank verpflichtet.

${ }^{8}$ Siehe v. Angerer, Wissenschaftliche Photographie [1931], S.145. Vergl. auch H. K i enle, Z. Physik 58, 726 [1929] und Z. Astrophysik I, 13 [1930].

\title{
Eine Deutung der Formierungserscheinungen bei Selen-Sperrschicht-Gleichrichtern ${ }^{1}$
}

\author{
Von Frank Rose und Hans Schmidt \\ (Z. Naturforschg. 2 a, 226-233 [1947]; eingegangen am 28. September 1946)
}

\begin{abstract}
In der vorliegenden Arbeit ist der Versuch unternommen worden, die bei der Formierung und Lagerung von Selen-Sperrschicht-Gleichrichtern auftretenden Erscheinungen durch eine Verlagerung von Störstellen in der Randschicht und ihre Abwanderung in die Deckelektrode zu erklären. Es wird gezeigt, daß unter diesen Voráussetzungen mit Hilfe der Schottky schen Randschichttheorie eine qualitative Behandlung der bei Versuchen aufgefundenen Effekte, von denen eine Anzahl diskutiert werden, möglich ist.
\end{abstract}

$\dot{\mathrm{U}} \dot{\mathrm{U}}$ ber die Arbeitsweise der Trockengleichrichter wurde von W. Schottky eine Theorie entwickelt ${ }^{2}$, die es unter der Annahme einer in ihrer Breite spannungsabhängigen Verarmungszone für Leitungs- (Defekt-) Elektronen an der Grenze Metall-Halbleiter gestattet, die Gleichrichterwirkung zu erklären. Hierbei wird angenommen, daß die Leitungselektronen durch Ionisierung praktisch unbeweglicher Fremdatome oder Moleküle, der sogenannten Störstellen im Halbleiter, gebildet werden. Diese Theorie kann durch Kapazitätsmessungen geprüft werden, die gleichzeitig die Möglichkeit bieten, die örtliche Verteilung der Störstellen am Rande des Halbleiters zu bestimmen.

Solche von uns ausgeführten Kapazitätsbestimmungen und Entladungsmessungen an SelenSperrschicht-Gleichrichtern lieferten den eindeutigen Beweis, daß die Beweglichkeit der dissoziierten Störstellen unter dem Einfluß angelegter Spannungen nicht $\mathrm{zu}$ vernachlässigen ist. Stör-

1 Entstanden im Herbst 1943 in Berlin. Z. Zt. Hamburg und Bonn. stellenbestimmungen ergaben z. B. eine eindeutige Veränderung der Störstellenverteilung durch Formierung (Abb. 1). In der vorliegenden Arbeit

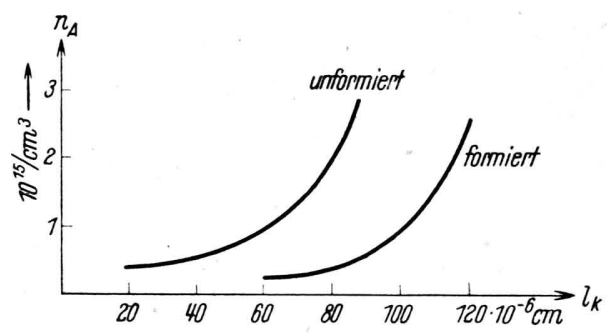

Abb. 1. Änderung der Störstellenverteilung durch Formierung.

haben wir daher den Versuch gemacht ${ }^{3}$, eine solche Störstellenverschiebung zur Deutung von Erscheinungen heranzuziehen, die bei der sogenannten Formierung, Lagerung und bei Strom-

2 W. Schottky, ,Vereinfachte und erweiterte Theorie der Randsohichtgleichrichter", Z. Physik 118, 9 u. 10 [1942].

${ }^{3}$ Ansätze zu einer Deutung wurden von W.Sch ot tk y auf Grund einer unveröffentlichten Arbeit von F. R o se gemacht. 
spannungsmessungen an Selen-Gleichrichtern auftreten.

Es hat sich hierbei herausgestellt, daß nicht nur zeitliche und spannungsbedingte Widerstandserhöhungen (Formierwirkungen) auftreten, sondern unter gewissen Bedingungen auch Erniedrigungen des Widerstandes (Antiformierwirkungen.) gefunden werden. Beide Effekte lassen sich im Rahmen der Schottkyschen Randschichttheorie durch Störstellenverlagerung erklären, der Formiereffekt durch Störstellenwanderung in Gebiete, wo diese unwirksam gemacht werden, z. B. durch Einwanderung in die Elektrode ${ }^{4}$ oder in eine dem Metall unmittelbar vorgelagerte Schicht, die Antiformierung dagegen durch Verlagerung von Störstellen innerhalb der Randschicht auf die Elektrode zu ohne merkliche Vernichtung derselben.

Es gelingt auf diese Weise, nicht nur wesentliche Züge des Formierungsvorganges, sondern auch andere, teilweise bekannte, aber unseres Wissens' bisher nicht gedeutete Erscheinungen qualitativ zu erklären.

\section{Formelmäßige Erfassung der notwendigen Ansätze}

a) Aus der Schottkyschen Theorie abgeleitete mathematische Voraussetzungen

Für das folgende sind einige der Sch ot th y schen vereinfachten Theorie der RandschichtGleichrichter entnommene Ansätze notwendig. Sie seien den weiteren Ausführungen vorangestellt und, soweit es nötig erscheint, erläutert.

Für die Stromstärke in einer Randverarmungsschicht gilt nach dieser Theorie unter Vernachlässigung der Bildkraft und wellenmechanischer Anteile bei Annahme einer örtlich homogenen Störstellenverteilung

$$
i=\varkappa_{R}\left(\mathfrak{E}_{R} \frac{1-e^{-U / \mathfrak{B}}}{1-e^{-\left(U+V_{D}\right) / \mathfrak{B}}} .\right.
$$

$\varkappa_{R}=e_{0} b n_{R}:$ Leitfähigkeit des Halbleiters am Metallrand

$b$ : Beweglichkeit der Defektelektronen

$n_{R}$ : Konzentration der Defektelektronen am Metallrand

4 Eine solche Überführung ron Störstellen in die Deckelektrode wird aus anderen Gründen auch von P. S e le ny i angenommen („A. Technikai Szelenogyeniranyiotek Elmeleteröl“, A. Magyar Elektrotechnikai Egyesület kiadasa, Budapest 1943). $e_{0}$ : Ladung des Defektelektrons

$\mathfrak{E}_{R}:$ Randfeldstärke

$\mathfrak{B}:$ Voltäquivalent der Versuchstemperatur

$V_{D}:$ Diffusionsspannung

$i$ : Stromstärke

$U$ : Anliegende Spannung.

Diese Gleichung gilt nach einer unveröffentlichten Arbeit von F. R o s e auch für örtlich inhomogene Störstellenverteilung, wenn $V_{D}$ durch ein Korrektionsglied erweitert wird.

Im Sperrgebiet vereinfacht sich diese Formel wegen $V_{D} \gg \mathfrak{B} \mathrm{zu}$ :

$$
i_{s p}=x_{R} \mathfrak{E}_{R}\left(1-e^{-U / \mathfrak{Z})} .\right.
$$

Betrachtet man bei einer bestimmten anliegenden Spannung den Sperrstrom vor und nach einer Veränderung der Randschicht, z. B. einer Verlagerung von Störstellen, so muß nach (1) für das Verhältnis der Ströme gelten:

$$
i_{1} / i_{2}=\mathfrak{E}_{R_{1}} / \mathfrak{E}_{R_{2}} .
$$

$\varkappa_{R}$, das nur von der Austrittsarbeit Metall-Halbleiter abhängt, soll dabei nicht verändert werden. Maßgebend für die Stromänderung ist in diesem Falle also allein das Verhältnis der Randfeldstärken. Der im folgenden durchgeführte Deutungsversuch beruht dementsprechend auf einer Beurteilung der durch Veränderungen der Randschicht, d. h. durch Störstellenverlagerung hervorgerufenen Feldstärkeänderungen. Die Deutung ist gültig für jeden theoretischen Ansatz, in dem die Feldstärke maßgebenden Einfluß auf den Sperrstrom hat. Es kann auch z. B. die Bildkraftwirkung mit . einbezogen werden.

Für den Feldstärkegradienten in der Randschicht, in der mit guter Annäherung die Raumladungsdichte

$$
\varrho=-e_{0} n_{A}
$$

gesetzt werden kann, gilt

$$
\frac{\partial \mathfrak{E}}{\partial x}=\frac{4 \pi \varrho}{\varepsilon}=-\frac{4 \pi e_{0}}{\varepsilon} n_{A}(x),
$$

d.h. die Feldstärke nimmt vom Metallrand $\left(\mathfrak{\Im}_{R}\right)$ nach dem Seleninnern zu ab. Da sie definitionsgemäß an der Randschichtgrenze $l_{k}$ null wird, ist die Gesamtfeldstärkeänderung in der Randschicht 


$$
\mathfrak{E}_{R}=\frac{4 \pi e_{0}}{\varepsilon} \int_{0}^{l_{k_{0}}} n_{A}(x) d x .
$$

Die Randfeldstärke $\mathfrak{F}_{R}$ läßt sich also aus der Störstellendichte $n_{A}(x)$ in der Randschicht, deren Verlauf neben dem der Defektelektronen in Abb. 2 ungefähr wiedergegeben ist, berechnen. Fordert man aus irgendwelchen Gründen in der Randschicht ein Konzentrationsgefälle der Leitungselektronen, so muß selbst im stromlosen Zustand, d.h. wenn von außen keine. Spannung angelegt wird, über der Randschicht ein elektrisches Potential $V_{D}$ vorhanden sein, welches einen Konzentrationsausgleich verhindert.

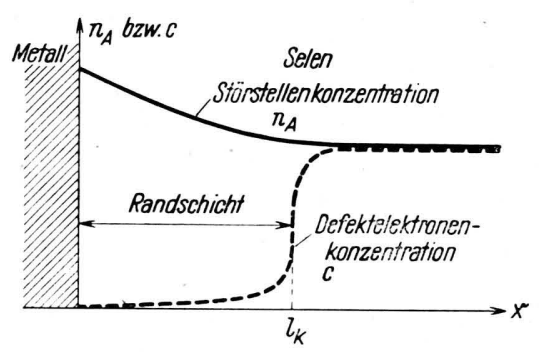

Abb. 2. Defektelektronen- und Störstellenverteilung (schematisch).

Dieses elektrische Diffusionspotential $V_{D}$ wird nach außen ohne Wirkung sein, da im Mittel die von ihm hervorgerufene Elektronenverschiebung durch den Elektronenstrom im Konzentrationsgefälle genau kompensiert wird.

Soll ein Strom fließen, so muß die über der Randschicht liegende Potentialdifferenz $V_{R}$ erhöht werden, so daß der Feldstrom größer als der Diffusionsstrom wird. Das kann man erreichen durch Anlegen einer äußeren Sperr-Spannung $U_{s p}$. Es gilt dann:

$$
V_{R}=V_{D}+U_{s p}
$$

Diese an der Randschicht liegende Spannung $V_{R}$ ist für die noch folgenden Überlegungen von Bedeutung. Sie ist einerseits gegeben durch (3), kann aber andererseits auch auf folgende Weise ausgedrückt werden:

Nach dem Vorausgegangenen ergibt sich, daß die Feldstärke an der Stelle $x$

$$
\mathfrak{F}_{x}=\mathfrak{F}_{R}-\frac{4 \pi e_{0}}{\varepsilon} \int_{0}^{x} n_{A}(x) d x=\frac{4 \pi e_{0}}{\varepsilon} \int_{x}^{l_{k}} n_{A}(x) d x
$$

ist. Durch Integration erhält man die an der Randschicht liegende Spannung

$$
\begin{aligned}
V_{R} & =\int_{0}^{l_{k}}\left(\mathcal{E} x ; d x \frac{4 \pi \rho_{0}}{\varepsilon} \int_{0}^{l_{k} \int_{k}} n_{A}(x) d x d x\right. \\
& =\frac{4 \pi e_{0}}{\varepsilon} \int_{0}^{l_{k}} x n_{A}(x) d x .
\end{aligned}
$$

b) Einflußeiner Störstellenverschiebung auf die Größe des Sperrstromes

Aus den oben abgeleiteten Formeln läßt sich der Einfluß einer Störstellenverschiebung auf die Größe des Sperrstromes zumindest qualitativ ableiten:

Wandern Störstellen von einer Stelle $x$ der Randschicht zum Metallrand $(x \rightarrow 0)$, ohne in die Deckelektrode überzugehen oder abgeschieden $\mathrm{zu}$ werden, so wird der Integrand von $V_{R}$ (4) um die Größe $x \cdot \Delta n_{A}$ verkleinert, wenn $\Delta n_{A}$ die Konzentrationsabnahme an der Stelle $x$ bedeutet.' Soll die anliegende Sperrspannung und damit $V_{R}$ erhalten bleiben, so muß die obere Grenze des Integrals (4) erweitert werden, d. h. die Randschicht dehnt sich aus, es werden zusätzlich Störstellen des neutralen Selens in dieselbe einbezogen. Die Verbreiterung bedeutet also eine Erhöhung der Gesamtstörstellenzahl in der betrachteten Randverarmungsschicht und damit eine Erhöhung der Randfeldstärke $\mathfrak{E}_{R}$, die ja nach (2) durch diese Zahl gegeben ist. Entsprechend nimmt nach (1) auch der Sperrstrom zu.

c) Einflußeiner Störstellenabwanderung in die Deckelektrode auf den Sperrstrom

Werden Störstellen, die sich ursprünglich in der Randschicht befanden, in die Deckelektrode übergeführt und dadurch oder auf irgendeine andere Weise unwirksam gemacht, so muß zur Aufrechterhaltung der Spannung ebenfalls eine Randschichtverbreiterung - wie im vorangehenden $\mathrm{Ab}$ schnitt - erfolgen. Es läßt sich aber zeigen, daß der. dadurch hervorgerufene Zuwachs an Störstellen nicht den eingetretenen Verlust ausgleichen kann, so daß nach (2) die Randfeldstärke absinkt.

Bedenkt man, daß für den Integranden von (4) das Produkt aus Störstellenkonzentration und Entfernung von der Deckelektrode, also die Größe $x \cdot n_{A}$ maßgebend ist, so erscheint diese Behauptung durchaus verständlich; denn durch die Randverbreiterung werden Störstellen in einer Entfernung 
$x>l_{k}$ wirksam gemacht, während die auswandernden Störstellen sich ursprünglich an Stellen $x<l_{k}$ befanden. Es wird aber in dem Produkt $x \cdot n_{\boldsymbol{A}}$ schon eine verhältnismäßig geringe Störstellenmenge iñ einer Entfernung $l_{k}$ eine größere Menge aus der Entfernung $x<l_{k}$ zur Aufrechterhaltung der Spannung ausgleichen können. Durch eine Überführung von ursprünglich in der Randschicht befindlichen Störstellen in die Deckelektrode ergibt sich also, dảß bei festgehaltener Spannung die Randfeldstärke $\mathfrak{夭}_{R}$ und damit der Sperrstrom absinken müssen. Dasselbe gilt naturgemäß auch für jede andere Art eines Unwirksammachens von Störstellen in den betrachteten Randschichten.

Dieselbe Wirkung wie eine Einwanderung von Störstellen in die Deckelektrode hat nach einer Bemerkung von Sch ot tky auch eine Einlagerung in die der Elektrode unmittelbar (innerhalb etwa $10^{-7} \mathrm{~cm}$ ) vorgelagerte Selenschicht, falls in dieser Schicht durch einen Anstieg der Störstellendichte so hohe Felder vorhanden sind, daß die Defektelektronen sie ungehindert passieren können. Diese Schicht ist dann gewissermaßen noch mit zur Metallelektrode zu rechnen, vermag aber ihre Störstellen mit der übrigen Randschicht ungehindert auszutauschen.

\section{Deutung von Formierungsvorgängen}

Änderungen der Strom-Spannungskennlinien von Selen-Gleichrichtern unter dem Einfluß angelegter Spannungen bezeichnet man allgemein als Formierung. Insbesondere wird hierunter das erstmalige Anlegen einer Sperrspannung an eine aus der Fertigung kommende Gleichrichterscheibe verstanden. Der Zweck einer solchen Formierung liegt im Erzielen von einigermaßen reproduzierbaren und größenmäßig brauchbaren Strom-Spannungswerten.

Die Vorgänge, die sich bei einer derartigen Formierung abspielen, kann man sich etwa folgendermaßen vorstellen:

Unter dem Einfluß einer angelegten Formier(Sperr-) Spannung wandern Störstellen der Randschicht auf die Deckelektrode zu und rufen vor ihr eine Erhöhung der Störstellendichte hervor, während in den weiter abliegenden Gebieten eine Verminderung der Konzentration gegenüber dem ursprünglichen Zustand auftritt. Hat die Konzentrationserhöhung vor der Elektrode einen gewissen Wert erreicht, so werden Störstellen in größerer Menge unwirksam. Dies kann man sich auf
Grund der hier bevorzugten Vorstellung so erklären, daß sie in das Metall übertreten, ohne hierbei chemisch gebunden zu werden. Man muß nämlich annehmen, daß zumindest eine gewisse Schicht des Metalles bereits mit solchen aus dem Selen stammenden Atomen abgesättigt und daher chemisch inaktiv ist. Diese Oberflächenschicht wird bei den weiteren Betrachtungen, wenn nicht besonders hervorgehoben, mit zur Metallelektrode gerechnet.

Mit Hilfe dieser durchaus nicht allein möglichen, aber verhältnismäßig anschaulichen Vorstellung sieht der Formierungsvorgang im einzelnen folgendermaßen aus: Im Gleichgewichtszustand wird einer gewissen Störstellenkonzentration im Selen eine bestimmte Störstellendichte in der oberflächlichen Metallschicht entsprechen. Wandern Störstellen, was sie schon ohne Anlegen einer äußeren Spannung unter dem Einfluß der Diffusionsspannung, die durch die Randverarmung der Defektelektronen hervorgerufen wird und nicht mit dem Konzentrationsgefälle der Störstellen verwechselt werden darf, tun werden, auf die Deckelektrode zu, so wird vor derselben eine gewisse Konzentrationserhöhung hervorgerufen und es werden Störstellen in die Elektrode übergehen.

Erhöht man die an der Randschicht liegende Spannung, so wird eine stärkere Konzentrationserhöhung in der Randschicht vor der Deckelektrode auftreten, ein Übergang einer größeren Anzahl von Störstellen in das Metall wird aber trotzdem nicht erfolgen. Wegen der geringen Diffusionsgeschwindigkeit wird nämlich im Metall ein Weiterwandern kaum erfolgen, so daß sich dort am Rand schon durch Übertritt sehr geringer Störstellenmengen die Konzentration ebenfalls stark vergrößern muß. Die Randkonzentrationen werden beiderseits so lange anwachsen, bis auf der Metallseite das Diffusionsgefälle derart groß ist, daß der Abtransport-merkbar wird und damit auch ein stärkerer Übergang von Störstellen in die Deckelektrode erfolgen kann (Abb.3). Dieser Übertritt von Störstellen in die Deckelektrode wird jedoch allmählich geringer werden, da mit der Zeit auf der Seite des Selens in der Randschicht durch Störstellenverarmung der entfernteren Gebiete sich so große Konzentrationsunterschiede ausbilden ${ }^{5}$,

5 Auf hohe Konzentrationsunterschiede in der Randschicht deuten Störstellenbestimmungen im Flußgebiet hin. 


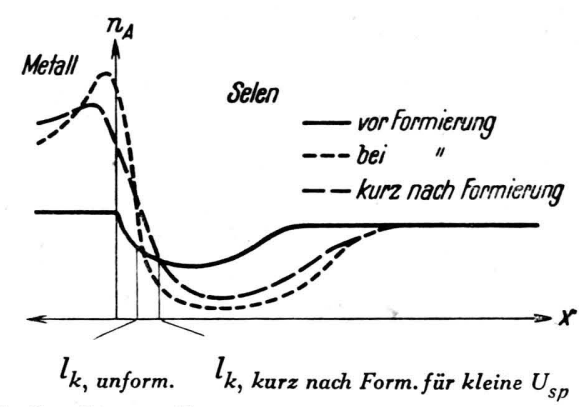

Abb. 3. Störstellenverteilungen (schematisch).

daß das Konzentrationsgefälle die Wirkung der Feldstärke ausgleicht, d.h. der Diffusionsstrom wird gleich dem Feldstrom. Die Störstellennachlieferung hört damit praktisch auf. Das Konzentrationsgefälle in der Randschicht kann allerdings durch Diffusion von Störstellen aus dem neutralen Selen verringert werden, was neben der Diffusion im Metall ein langzeitiges Weiterlaufen des Formierungsprozesses mit geringer Wirksamkeit ermöglicht.

Wird die Spannung abgeschaltet, so liegt an der Randschicht wieder nur die Diffusionsspannung. Die Störstellen werden daher im Konzentrationsgefälle zurücklaufen und es wird sich eine Dichteverteilung im Selen einstellen, die ähnlich derjenigen vor der Formierung ist (Abb. 3). Ein grundsätzlicher Unterschied besteht jedoch: Wegen des erfolgten Überganges von Störstellen in die Deckelektrode muß die Gesamtzahl der Störstellen in der Randschicht jetzt geringer sein. Dementsprechend muß auch die Verteilungskurve bei ähnlicher Form tiefer liegen. Ein Rücktransport von Störstellen aus der Deckelektrode in die Randschicht wird zwar ebenfalls erfolgen, jedoch wegen der geringen Diffusionsgeschwindigkeit im Metall beachtlich langsamer vor sich gehen.

Bei allen hier geschilderten Prozessen wird im übrigen ein merkbarer Einfluß der Temperatur vorhanden sein, da diese für die Größe der Diffusion und Beweglichkeit mitbestimmend ist.

Der alte Zustand, der vor der Formierung vorlag, kann sich durch Rückwanderung von Störstellen in das Selen aus dem eben angeführten Grunde erst allmählich wieder herstellen. Eine völlige Rückbildung der alten Verhältnisse wird jedoch nicht mehr eintreten, wenn ein Teil der in der Oberflächenschicht des Metalles eingewanderten Störstellen durch ein starkes Konzentrationsgefälle Gebiete erreicht, in denen die Metallatome noch nicht chemisch abgesättigt sind. Dort werden dann Störstellen gebunden, d. h. fest in das Metallgitter eingelagert werden, für die damit eine Rückdiffusion unmöglich geworden ist.

\section{Erklärung beobachteter Erscheinungen}

Mit der soeben eingehend beschriebenen Vorstellung einer Wanderung von Störstellen unter dem Einfluß von Sperrspannungen und ihrer Überführung in die Deckelektrode läßt sich ein großer Teil der bei der Formierung und Lagerung von Gleichrichtern auftretenden Effekte erklären.

\section{A. Formierung}

Mißt man den während einer Formierung bei konstanter Spannung durch den Gleichrichter fließenden Strom, so zeigt sich nach einem kurzen Anstieg ein asymptotisches Abklingen desselben (Abb.4). Dieser Stromverlauf ist nach dem Vorausgegangenen verständlich. Solange nämlich die Wanderung von Störstellen auf die Deckelektrode $\mathrm{zu}$, hervorgerufen durch eine anliegende Formierspannung, dort nur eine Konzentrationserhöhung ohne merklichen Utbertritt in das Metall bewirkt, muß nach den obigen Ausführungen die Stromstärke wachsen. Erst wenn bei annähernd gleichbleibender Randkonzentration die Abwanderung

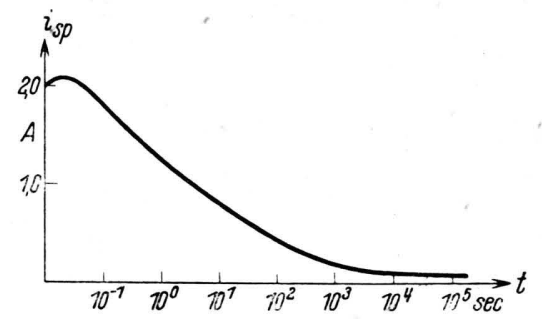

Abb. 4. Zeitlicher Verlauf des Stromes bei einer Formierung.

größerer Störstellenmengen in die Deckelektrode erfolgt, darf der Strom absinken. Wegen des allmählichen Abklingens des gesamten Verlagerungsvorganges muß sich die Stromabnahme, wie dies auch tatsächlich der Fall ist, asymptotisch einem Endwert nähern.

\section{B. L a ger ung}

Wird die Formierspannung abgeschaltet, so sollte, wie eben gezeigt, nach weitgehender Wiederherstellung der ursprünglich in der Rand- 
schicht vorliegenden Konzentrationsgradienten eine langsame Rückdiffusion von Störstellen aus der Deckelektrode in das Selen erfolgen. Ein solcher Vorgang muß aber eine zeitlich entsprechend ausgeführte Wiedererhöhung des Sperrstromes hervorrufen. Diese Erscheinung wird auch wirklich gefunden und ist als Sperrstromlageralterung bekannt (Abb.5).

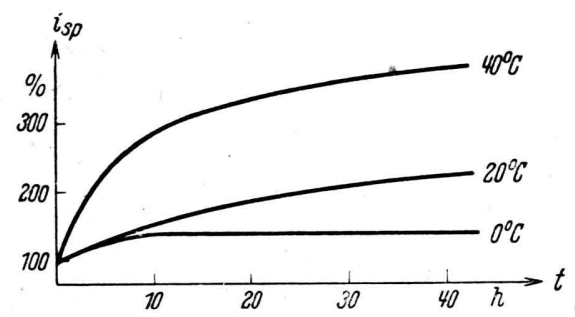

Abb. 5. Anstieg des Sperrstromes bei fester Meßspannung nach Lagerung bei verschiedener Temperatur.

Daß die Alterung temperaturabhängig ist, ist verständlich, denn bei hoher Temperatur wird die Diffusion im Metall und damit die Rückwanderung ins Selen schneller erfolgen als bei niedriger. Daß auch dieser Prozeß asymptotisch einem Endwert zustrebt, der dem Zustand vor der Formierung aber nicht völlig entspricht, ergibt sich ohne weiteres aus den Vorstellungen über das Verhalten der Störstellen nach Abschalten der Formierspannung (Abschnitt 2).

C. Verschiedene bei Messungen auftretende Effekte

a) Stromerhöhung durch die Formierung bei niedriger Meßspannung: Mißt man kurz nach einer Formierung den Sperrstrom bei niedrigen Spannungen (bis 1,5 Volt), so erhält man höhere Stromwerte als im unformierten Zustand. Bei hohen Meß-Spannungen ergibt sich dagegen, wie gezeigt, ein umgekehrtes Verhalten.

Diese Erscheinung läßt sich ebenfalls erklären: Wenn auch die Störstellenbeweglichkeit im Selen beachtlich größer ist als im Metall, so kann man doch annehmen, daß innerhalb der Randschicht nach Abschalten der Formierspannung die Rückwanderung der Störstellen dann, wenn der Gleichgewichtszustand fast erreicht ist, sehr langsam vor sich geht, insbesondere bei nicht sehr hoher Formierungstemperatur. Es ist daher, wie. in Abb. 3 schematisch gezeigt, durchaus möglich, daß in den der Deckelektrode sehr nahen Gebieten der
Störstellengehalt höher ist als vor der Formierung. Da aber bei niedrigen Sperrspannungen die Randschichtbreite gering ist, erfaßt die Messung dann nur ein schmales, an die Deckelektrode angrenzendes Gebiet. In diesem kann, zumindest kurz nach der Formierung, der Gesamtstörstellengehalt größer sein als vorher, womit der Anstieg des Sperrstromes bei kleinen Meß-Spannungen erklärt ist.

Mißt man dagegen bei einer Sperrspannung, die von gleicher Größenordnung ist wie die Formierspannung, so ist das dann betrachtete Gebiet annähernd gleich der Randschicht bei der Formierung. In diese erfolgte aber durch den Formierungsprozeß praktisch keine Einwanderung, sondern es fand in ihr nur eine Verlagerung und Abwanderung statt, so daß sich für die bei hohen Meß-Spannungen vorliegende Sperrschicht ebenso wie für die bei der Formierung vorhandene Randverarmungszone eine Abnahme der Störstellenzahl und damit ein Sinken des Sperrstromes ergibt .

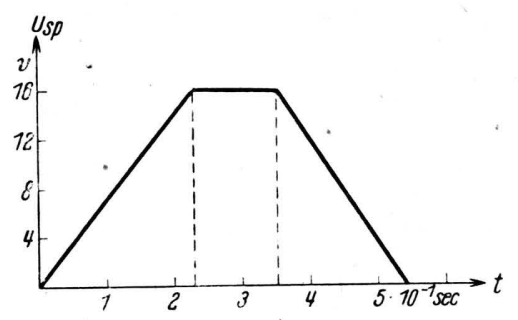

Abb. 6. Zeitlicher Verlauf der Meßspannung.

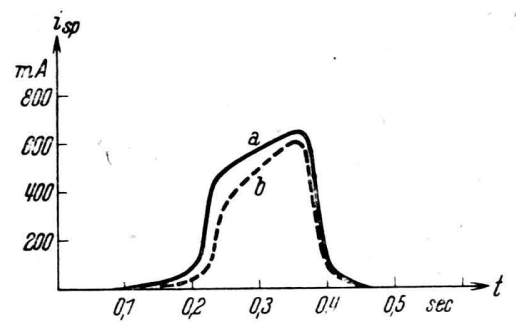

Abb. 7. Sperrstromverlauf 1,5 Stdn. nach einer Formierung.

b) Stromverlauf bei Anlegen einer trapezförmig verlaufenden Gleichspannung: Legt man kurz (ca. 1,5 Stdn.) nach einer Formierung bei $12 \mathrm{Volt}$ eine zeitlich trapezförmig verlaufende Sperrspannung (Abb. 6) mit einem Maximalwert von 16 Volt an einen Selengleichrichter, so weist der Sperrstrom den in Abb. 7 (Kurve $a$ ) wiedergegebenen Verlauf auf. Selbst im Gebiet konstanter Span- 
nung ist hier ein zeitliches Anwachsen der Stromstärke zu bemerken.

Dieses Verhalten läßt sich folgendermaßen deuten:

Nach Abschalten der Formierspannung hat sich die Konzentrationsüberhöhung vor der Deckelektrode durch Rückdiffusion teilweise ausgeglichen. Bei Einschalten der Meß-Spannung wird sie sich von neuem ausbilden. Es erfolgt also eine Störstellenwanderung auf die Deckelektrode zu, die sich durch einen Stromanstieg bei konstanter Spannung bemerkbar macht.

Ist, wie im vorliegenden Fall, die Meß-Spannung größer als die vorher verwandte Formierspannung, so erfolgt durch die Messung selbst eine Nachformierung. Diese ruft bei einem zeitlich nicht sehr ausgedehnten Vorgang nur eine Verflachung des Stromanstieges hervor: es tritt dann nämlich zwar schon eine merkbare Überführung von Störstellen in die Deckelektrode auf, es erfolgt aber gleichzeitig noch ein Konzentrationsanstieg in den vor ihr liegenden Randschichtgebieten.

Bei einer zweiten Messung muß die Stromstärke geringer sein, weil durch die Abwanderung in die Deckelektrode der Störstellengehalt gesunken ist. Außerdem darf keine so ausgeprägte Verflachung des Stromanstieges mehr gefunden werden, da der weitere Übertritt von Störstellen in die oberflächlich gesättigte Deckelektrode erschwert ist. Beide Forderungen konnten experimentell bestätigt werden (Abb. 7, Kurve b).

Nach längerer Lagerung wird eine weitgehende Rückwanderung von Störștellen aus dem Metall in das Selen stattgefunden haben. Es ist dann durch die Messung wieder eine Formierung zu erwarten, so daß der Stromverlauf dem dort vorliegenden ähnlich sein muß, d. h. das Gebiet des Anstieges bei konstanter Spannung ist weitgehend überlagert durch ein abwanderungsbedingtes $\mathrm{Ab}$ sinken des Sperrstromes. Je nach der Dauer der Lagerung werden Übergänge von der einen zur anderen Kurvenform zu erwarten sein. Vorgenommene Messungen weisen dieses Verhalten nach (Abb. 8 und 9).

c) Intermittenz-Effekt: Schaltet man bei der Formierung eines Sperrschicht-Gleichrichters kurzzeitig die anliegende Sperrspannung ab, so nimmt bei Wiedereinschalten der Strom nicht sofort seinen alten Wert an, sondern ,kriecht“ in

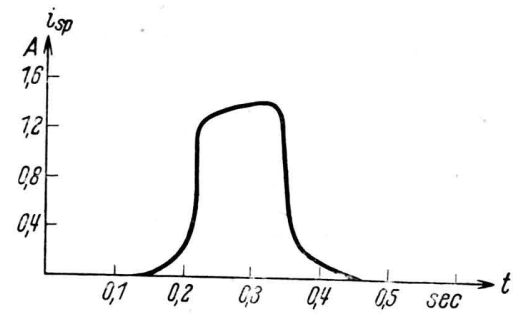

Abb. 8. Sperrstromverlauf 40 Stdn. nach einer Formierung.

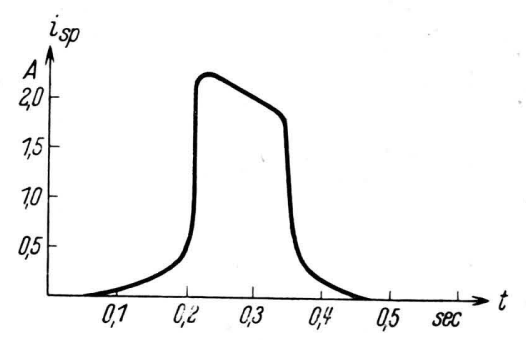

Abb. 9. Sperrstromverlauf 240 Stdn. nach einer Formierung.

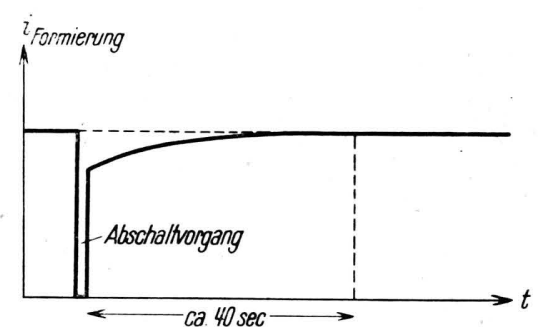

Abb. 10. „Kriechen“ des Stromes nach kurzzeitigem "Abschalten bei einer Formierung.

einer Zeit von etwa 30 bis 40 Sek. auf denselben zurück (etwa wie in Abb. 10 dargestellt).

Diese Erscheinung kann man so erklären: Vor dem Abschalten der Spannung besteht innerhalb der Randschicht annähernd Gleichgewicht zwischen der elektrischen Feldstärke und dem Diffusionsgefälle. Schaltet man die Sperrspannung ab, so wird die Feldstärke auf einen Bruchteil, der dem von der Diffusionsspannung hervorgerufenen Anteil entspricht, verringert und die Störstellen stehen praktisch unter dem Einfluß eines sehr starken Diffusionsgefälles. Bei der durch die Formierung bedingten hohen Temperatur wird daher ein anfänglich sehr starker Konzentrationsausgleich erfolgen. Legt man die Spannung nach so kurzer Zeit wieder an, daß trotzdem nur eine sehr geringe Änderung der Konzentrationsverhältnisse, z. B. um $10 \%$, eingetreten ist, so ist nach dem Wie- 
dereinschalten das Gleichgewicht nur um diese Abweichung gestört. Die dann auf die Störstellen wirkende resultierende Kraft beträgt im Falle unseres Beispieles nur 10\% derjenigen, welche bei Abschalten die Störstellenverlagerung hervorrief. Die Rückbildung der alten Verhältnisse beim Wiederanlegen der Spannung wird daher beachtlich langsamer vor sich gehen als der beim $\mathrm{Ab}$ schalten einsetzende Prozeß.

Der Vorgang ist vergleichbar mit einer teilweisen Entladung eines Kondensators und anschließender Wiederaufladung. Bei der Entladekurve befindet man sich hierbei im Gebiet großer Steilheit, während für die Wiederaufladung nur

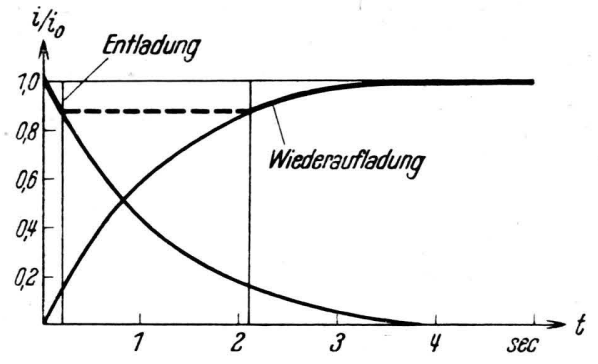

Abb. 11. Kurzzeitige Entladung mit anschließender Wiederaufladung eines Kondensators.

der flach ansteigende Teil der Kurve maßgebend ist (Abb. 11).

\title{
Gasballastpumpen
}

\author{
Von Wolfgang Gaede $\dagger 1$ \\ (Z. Naturforschg. 2 a, 233-238 [1947]; eingegangen *am 1. Oktober 1946)
}

\begin{abstract}
Beim Absaugen von dampfhaltigen Gasen kondensiert sich ein Teil des Dampfes während des Kompressionsvorgangs in der Pumpe. Dies kann durch Einlassen von zusätzlicher Luft, „Gasballast“ genannt, in den Schöpfraum der Pumpe vermieden werden. Die Arbęitsweise derartiger Pumpen wird beschrieben, die zur Vermeidung von Kondensation erforderlichen Gasballastmengen werden ermittelt.

Das erforderliche Gasballastvolumen ist um so niedriger, je geringer der Partialdruck des Dampfes im angesaugten Gase ist; daher ist die Einschaltung eines Kondensators vor der Pumpe wichtig. Über dem Kondensator ist das Verhältnis der Partialdrucke von Gas und Dampf gegenüber dem freien Gasraum verschoben, so daß eine Anreicherung von Gas über der Kondensationsfläche eintritt, die „Gasschleier“ genannt wird. Ein experimenteller Nachweis dieses Gasschleiers, der von grundsätzlicher Bedeutung für die Betrachtung von Kondensationsvorgängen in dampfhaltigen Gasen ist, wird beschrieben.
\end{abstract}

1. Wirkungsweise der Gasballast$\mathrm{p} \mathrm{u} \mathrm{m} \mathrm{p} \mathrm{e}$

$\mathrm{D}$ ie rotierenden Ölluftpumpen verdanken ihre gr@ßeVerbreitung ihrer Unverwüstlichkeit und dem erreichbaren hohen Endvakuum, solange sie nur zum Absaugen von Luft verwendet werden. Beim Absaugen von Dämpfen, beispielsweise bei

1 Im März 1944 reichte Wo l f g a n g G a e d e bei der Zeitschrift für technische Physik ein Manuskript „Gasballastpumpen und Vakuumtechnik des Dampfes“ ein, in dem die Verhältnisse in dem Druckbereich zwischen 20 und 0,01 Torr, insbesondere auch bei dampfhaltigen Gasen, in ähnlich gründlicher Weise untersucht wurden, wie dies in früheren Veröffentlichungen über das eigentliche Hochvakuumgebiet erfolgt ist. Bei Kriegsende lag die druckfertige Korrektur vor, die ihres Umfanges wegen z. Zt. aber nicht publiziert werden kann. - Die vorliegende Ver- einer Vakuumdestillation, versagt. die Ölluftpumpe jedoch nach kurzer Betriebszeit. Die Dämpfe kondensieren bei dem Kompressionsvorgang innerhalb der Pumpe, und das Kondensat verunreinigt das Pumpenöl. Die in dieser Abhandlung beschriebenen Gasballastpumpen sind rotierende Ölluftpumpen, die gegen das Absaugen von Dämpfen unempfindlich gemacht sind. Dies

öffentlichung stellt einen Auszug aus dem Manuskript dar. Bei seiner Anfertigung habe ich mich auf die Abschnitte mit vorwiegend physikalischem Inhalt beschränkt. Die außerdem im ursprünglichen Manuskript enthaltene ausführliche Diskussion der Maßeinheiten der Vakuumtechnik, die Ausführungen über die Druckmessungen in Dampf-Gas-Gemischen, eine Anzahl Experimente zum Nachweis des Gasschleiers sowie sämt. liche Kapitel rein technischen Inhalts müssen einer späteren Veröffentlichung vorbehalten bleiben. M. Dunkel, St. Andreasberg (Harz). 